\title{
Traduire
}

Ine autre perspective sur I t tadaduction

Revue française de la traduction

$236 \mid 2017$

Traducteurs en blouse blanche

\section{La traduction médicale : des nomenclatures à l'orthographe, petit florilège des pièges et difficultés}

Nathalie Renevier

\section{(2) OpenEdition \\ Journals}

Édition électronique

URL : http://journals.openedition.org/traduire/907

DOI : 10.4000/traduire.907

ISSN : 2272-9992

Éditeur

Société française des traducteurs

Édition imprimée

Date de publication : 1 juin 2017

Pagination : 5-12

ISSN : 0395-773X

\section{Référence électronique}

Nathalie Renevier, «La traduction médicale : des nomenclatures à l'orthographe, petit florilège des pièges et difficultés », Traduire [En ligne], 236 | 2017, mis en ligne le 01 juin 2019, consulté le 10 décembre 2020. URL : http://journals.openedition.org/traduire/907 ; DOI : https://doi.org/10.4000/ traduire.907 


\section{La traduction médicale : \\ des nomenclatures à l'orthographe, petit florilège des pièges et difficultés}

\section{Nathalie Renevier}

Comme tout domaine de spécialité, la médecine a son propre langage, souvent abscons pour le commun des mortels. Et c'est bien là sa première difficulté. En effet, chacun d'entre nous sera un jour confronté au jargon médical. Que ce soit pour une simple consultation afin d'obtenir un certificat d'aptitude à la pratique d'un sport, pour une toux qui ne passe pas, un suivi de grossesse, une entorse ou quelque chose de bien plus grave, nous serons tous amenés à nous rendre chez le médecin au moins une fois dans notre vie. Et il n'est pas rare que la terminologie complexe à laquelle nous sommes confrontés nous fasse craindre le pire, heureusement souvent sans raison.

Ainsi, le grand public a besoin de comprendre et d'être rassuré. Il est donc essentiel de lui expliquer avec des mots simples les symptômes, diagnostics, examens et autres traitements, mais aussi de lui présenter les structures anatomiques et biologiques concernées de manière accessible. Une maman à qui on annonce aux urgences que son fils présente " une fracture de l'ulna sur le tiers distal de la diaphyse " risque fort d'être interloquée. En revanche, si le radiologue lui parle d'une "fracture du cubitus à proximité du poignet ", elle sera vite rassurée et comprendra immédiatement que trois semaines à un mois de plâtre suffiront à son enfant pour se rétablir. Ulna et cubitus désignent la même chose, l'os externe de l'avant-bras. S'ils sont parfaitement interchangeables du point de vue du sens, ces deux termes s'adressent à des publics différents, le premier appartenant à la Terminologia anatomica (TA), nomenclature anatomique internationale destinée aux spécialistes, le second à la nomenclature grand public, que tout le monde ou presque connaît.

Cette différence de niveau de langue entre deux publics amenés à se rencontrer régulièrement est l'une des principales difficultés auxquelles est confronté tout traducteur médical, car jongler avec les nomenclatures implique de les connaître sur le bout des doigts (ou à défaut d'avoir les bons outils pour faire les vérifications nécessaires). Ainsi, la TA, la CIM (Classification Internationale des Maladies) 10, et bientôt 11, ou les DCl (Dénominations Communes Internationales des molécules pharmaceutiques), pour n'en citer que quelques-unes, ne doivent avoir aucun secret pour lui. Et naturellement, afin d'éviter les incohérences, pas question 
d'alterner entre la nomenclature grand public et celle destinée aux spécialistes dans un même texte. II faut faire un choix et s'y tenir !

Arrêtons-nous sur la Terminologia Anatomica. Sa difficulté découle de son application très tardive en France. En effet, dès la fin du xıxe siècle naît la Basiliensa Nomina Anatomica (BNA), nomenclature latine établie par des anatomistes allemands. L'objectif est alors d'homogénéiser les dénominations, d'harmoniser les termes d'anatomie humaine et animale, et d'instaurer une certaine cohérence avec les termes existants.

Rapidement reconnue dans plusieurs pays européens et outre-Atlantique, la BNA n'est cependant pas adoptée par les francophones qui lui préfèrent la nomenclature classique. Malgré tout, après des années de résistance, au début des années 1950, une traduction visant à franciser la BNA est entreprise par des comités internationaux d'anatomie. La Parisiensa Nomina Anatomica paraît en 1955, suivie en 1956 par la première édition de la Nomina Anatomica (NA) qui contient déjà 5640 termes francisés. Le processus est bien lancé et 1989 marque la parution de la 6 e édition de la NA. Cependant, les principaux ouvrages d'anatomie français résistent encore et toujours à l'envahisseuse, et des atlas réputés, comme le Rouvière/ Delmas en quatre tomes, sont réédités avec l'ancienne nomenclature.

Dans les années 1990, le Comité fédératif international de terminologie anatomique ou FCAT (pour Federative Committee on Anatomical Terminology) lance une ultime révision de la NA, et en 1998, la Fédération internationale des associations d'anatomistes publie la version actuelle, qui s'appelle désormais la Terminologia Anatomica ou TA. Organisée en 16 systèmes, riche de plus de 7500 termes, la TA s'impose peu à peu, mais il faudra attendre le début des années 2000 pour que les atlas d'anatomie l'intègrent enfin. Entièrement revu et mis à jour, le Rouvière/ Delmas qui paraît en décembre 2002 est l'un des premiers à utiliser la TA.

Si les étudiants en médecine apprennent désormais l'anatomie version TA, face aux patients l'ancienne nomenclature, seule connue du grand public... et de très nombreux médecins en exercice depuis de longues années, reste de mise. Lors de colloques médicaux, combien de fois ai-je entendu des médecins, généralistes ou spécialistes, formés avant l'apparition de la $\mathrm{TA}$, se plaindre de ces changements ? Une amie médecin généraliste qui suit une formation en échographie me faisait récemment part de ses difficultés à retenir les "nouveaux termes ", notamment les noms des muscles. Et cela se comprend! Nous avons environ 650 muscles, dont plus de la moitié, pour ne pas dire les deux tiers, ont soit une nouvelle appellation francisée basée sur le latin, soit un nom latin dont la graphie doit être francisée !

Prenons les muscles fessiers, que tout le monde situera facilement. En latin gluteus, dans la TA, glutéal/glutéaux. Le muscle grand fessier, en latin gluteus maximus, est donc le muscle grand glutéal, le moyen fessier et le petit fessier (gluteus medius et gluteus minimus, respectivement), sont, en toute logique, le moyen glutéal et petit glutéal. Le muscle couturier (dans la cuisse), a pris son appellation latine de muscle sartorius, et en cas de crampe au mollet, il vous faudra étirer non plus les muscles jumeaux mais les muscles gastrocnémiens. Pas évident. 
Et les muscles sont loin d'être les seuls à avoir ainsi changé de nom. De multiples veines, artères, nerfs ou autres vaisseaux lymphatiques ont été renommés. Tous les ganglions sont devenus des nœuds, certains conduits sont aujourd'hui des canaux, et inversement ! Les trous sont désormais des foramens, les méats, des ostiums, les sommets, des apex, et certains organes ont été latinisés, comme l'iléon devenu l'iléum, qui forme l'intestin grêle avec le duodénum et le jéjunum. Le liquide céphalorachidien (LCR) devient cérébrospinal (LCS), la moelle épinière est maintenant spinale, et la colonne vertébrale est devenue le rachis. N'oublions pas non plus les os. La rotule, le cubitus, l'omoplate ou le péroné, pour ne citer que les plus connus, ont cédé la place à la patella, l'ulna, la scapula et la fibula, respectivement, avec au passage un changement de genre pour certains d'entre eux. Un véritable inventaire à la Prévert.

Enfin, dans la TA, la tendance est à la suppression des éponymes (noms propres). Ainsi, les trompes d'Eustache sont renommées trompes auditives, les trompes de Fallope sont remplacées par les trompes utérines, le canal de Santorini est désormais le conduit pancréatique accessoire, et les aires de Wernicke et de Broca, grâce auxquelles nous comprenons ce qu'on nous dit et nous nous faisons comprendre, ont été renommées aire de perception des mots et des symboles du langage et aire motrice du langage, respectivement. Là encore, la liste est loin d'être exhaustive.

Outre les noms des différentes structures anatomiques de base, la TA modifie les appellations relatives aux positions anatomiques. Quelques exemples à retenir, leur utilisation étant désormais systématique dès que l'on s'adresse à un spécialiste du domaine médical.

\begin{tabular}{|l|l|l|}
\hline \multicolumn{1}{|c|}{ Ancien terme } & Nouveau terme & \multicolumn{1}{c|}{ Exemple TA } \\
\hline $\begin{array}{l}\text { Supérieur (pour le segment d'un } \\
\text { membre ou une portion d'un } \\
\text { organe) }\end{array}$ & Proximal & Tiers proximal du tibia \\
\hline Supérieur (direction) & Crânial & Branche crâniale du pubis \\
\hline $\begin{array}{l}\text { Inférieur (pour le segment d'un } \\
\text { membre ou une portion d'un } \\
\text { organe) }\end{array}$ & Distal & Portion distale de l'intestin grêle \\
\hline Inférieur (direction) & Caudal & Segment caudal de la lingula \\
\hline Sous & Sub & Artère subclavière \\
\hline Sous & Infra & Muscle infraépineux \\
\hline Sus & Supra & Muscle suprascapulaire \\
\hline Interne & Médial & Muscle droit médial de l'œil \\
\hline Externe & Latéral & Muscle gastrocnémien latéral \\
\hline
\end{tabular}


Lors de la traduction d'un article médical, le traducteur doit donc impérativement demander à qui est destiné le texte cible afin d'utiliser la bonne nomenclature, car si un médecin s'adaptera facilement à un article destiné au grand public, ce dernier risque d'avoir du mal à comprendre le jargon du spécialiste.

Autre difficulté à laquelle le traducteur médical est régulièrement confronté : les abréviations, sigles et acronymes, dont la liste, quelle que soit la langue, est interminable. Sachant qu'il n'est pas rare qu'un même sigle ait plusieurs significations, y compris dans un même domaine, voire dans un même sous-domaine, le traducteur devra alors soigneusement en vérifier la signification et s'assurer qu'il n'y a pas de confusion possible.

Prenons par exemple le sigle AA. En anglais dans le domaine de la cardiologie, il peut signifier aortic aneurysm, abdominal aorta, ascending aorta, aortic amplitude ou aortic arch et en addictologie, active alcoholic, alcohol abuse ou alcoholics anonymous. Mais AA a aussi bien d'autres sens comme acetabular anteversion, acupuncture analgesia, acute appendicitis, adrenocortical autoantibody, acetic acid ou aplastic anemia, pour n'en citer que quelques-uns. Le Jablonski's Dictionary of Medical Acronyms and Abbreviations répertorie pas moins de 40 significations possibles pour ce seul sigle, uniquement dans le domaine médical ! Autant dire que certains types de documents - dossiers médicaux, comptes rendus opératoires ou résultats d'analyse - peuvent vite devenir de véritables casse-tête si le traducteur ne maîtrise pas le sujet.

Pour corser le tout, il n'est pas rare que le français conserve l'abréviation anglaise. Citons par exemple le LDL cholesterol (LDL = low density lipoprotein) traduit par cholestérol LDL à l'attention des professionnels de santé, et plus connu du grand public sous le nom de mauvais cholestérol. Le français (comme la plupart des autres langues d'ailleurs) garde LDL pour désigner les lipoprotéines de basse densité, dont l'abréviation française, qui serait plutôt LBD, n'est pas usitée. De plus, il arrive que par convention ou par habitude, un sigle anglais soit utilisé quelles que soient les langues source et cible, ou qu'un texte rédigé en espagnol conserve un sigle anglais, là où le français et l'allemand préféreront l'adapter. Au traducteur de faire le bon choix en toute connaissance de cause.

Enfin, les textes sources, notamment anglais, contiennent fréquemment des abréviations latines dont la graphie varie, comme q.d., qd ou QD pour quaque die, soit une fois par jour, b.i.d., bid ou BID, pour bis in die, soit deux fois par jour, p.r.n., prn ou PRN pour pro re nata, qui signifie si besoin, q.h., qh, QH pour quaque hora, soit toutes les heures, etc. La liste est longue, et le traducteur médical qui bute sur une abréviation doit avoir le réflexe de penser au latin s'il ne trouve rien qui corresponde dans la langue source.

Autre problème auquel il peut être confronté, même si ce n'est pas le plus fréquent, la polysémie, qui ne concerne pas uniquement les sigles. Pour ne citer qu'un exemple, peu connu, en anatomie, le trapèze désigne à la fois un muscle du dos composé de trois faisceaux et un os du carpe qui joue un rôle essentiel dans la mobilisation du pouce. Si à l'image du français, 
certaines langues, comme l'espagnol avec trapecio, désignent l'os et le muscle par le même terme, obligeant le lecteur à tenir compte du contexte pour savoir de quoi il retourne, d'autres emploient deux termes différents, comme l'anglais avec trapezius pour le muscle et trapezium pour l'os.

À ces difficultés déjà nombreuses, s'ajoute la proximité sémantique ou fausse synonymie dont le domaine médical regorge, l'anatomie étant un peu l'exception qui confirme la règle. Prenons les termes anglais wound et injury, qui de prime abord ne sont pas les plus difficiles à comprendre. Leur traduction en français, loin d'être figée, dépendra du contexte. Blessure, écorchure, égratignure, lésion, plaie, voire ulcère, font partie des solutions possibles. Mais si dans le langage courant, à l'exception d'ulcère, ces termes semblent plus ou moins interchangeables, dans la réalité médicale ils sont loin de l'être et l'utilisation de l'un à la place de l'autre est souvent incorrecte. Seul lésion, qui désigne l'altération morphologique d'un tissu ou d'un organe, convient dans la plupart des cas. Et si une plaie (rupture accidentelle de la barrière cutanée présentant un risque de souillure ou d'infection) est souvent une blessure, de nombreuses blessures (lésions traumatiques provoquées par un agent extérieur physique ou chimique), comme une fracture du tibia non ouverte ou une rupture (éclatement) de la rate sans lésion de la barrière cutanée, ne sont pas des plaies. De même, si une écorchure (lésion superficielle due à une abrasion ou un frottement) et une égratignure (lésion superficielle provoquée par un élément acéré ou pointu) sont des plaies, toutes les plaies ne sont malheureusement pas aussi superficielles qu'une écorchure ou une égratignure.

Autre exemple sur lequel on pourrait passer des heures, le terme anglais condition, qui, loin d'être aussi simple qu'il n'y paraît, est souvent mal traduit. En français, son équivalent strict condition doit être proscrit. Un patient ne souffre pas d'une condition. II peut être atteint d'une affection, d'une maladie, d'un trouble, d'un syndrome, d'un désordre, d'une infirmité, faire un malaise, une crise (d'angoisse, d'épilepsie), une poussée (de polyarthrite, de sclérose en plaques, d'eczéma) et bien d'autres choses encore, mais en aucun cas ici le traducteur français ne doit utiliser condition. II doit s'interroger sur le sens précis de chacun de ces termes, en rechercher la définition et évaluer les différentes possibilités dans son contexte avant de choisir celui qui convient le mieux. Attention, le terme pathologie [du grec pathos, souffrance, et logos, connaissance, étude], trop souvent utilisé au sens de maladie pour traduire notamment l'anglais pathology, mais aussi sickness, disease ou illness, désigne exclusivement la " science qui étudie les maladies ". Dire que "la grippe est une pathologie virale " ou que "le patient souffre d'une pathologie grave " est incorrect.

Les exemples choisis pour illustrer le problème de proximité sémantique ne sont qu'une goutte d'eau dans l'océan de la terminologie médicale, où l'utilisation du bon terme est parfois d'autant plus difficile que l'usage courant est erroné. Le terme anglais side effect est ainsi souvent traduit par effet secondaire. Or, ce terme est impropre. Selon le cas, on parlera plutôt d'effet indésirable (phénomène observé pendant l'étude clinique et dont on est sûr qu'il est dû au 
principe actif), d'événement indésirable (phénomène observé pendant l'étude clinique mais qui n'est pas nécessairement induit par le principe actif) ou encore d'événement secondaire (phénomène observé après la mise sur le marché, et qui n'est pas forcément nocif/indésirable). Le traducteur devra donc savoir à quel moment survient ce side effect pour bien le traduire et dans tous les cas, éviter les effets secondaires.

Une fois venu à bout de toutes ces difficultés, reste l'ultime étape de la relecture. Certes, une orthographe correcte n'est pas l'apanage des textes médicaux, mais certaines recommandations de l'Académie française compliquent le travail.

Tout d'abord, l'accentuation systématique des " e " dans les termes latins comme iléum, duodénum ou jéjunum dont nous avons déjà parlé, mais aussi placébo, nocébo ou sénior. Après des années passées à supprimer les accents lors des relectures, il faut désormais les mettre ! J'avoue que j'ai un peu de mal.

Ensuite, la francisation du pluriel des mots latins par l'ajout d'un " s " si nécessaire. II conviendra donc d'écrire des foramens (et non des foramina), des stimulus (au lieu de stimuli), des minimums (et non minima), etc.

Et enfin, dernière recommandation de l'Académie française, et non des moindres, la suppression du trait d'union sauf en cas de hiatus, ce qui est à l'origine de certaines bizarreries. Prenons quelques exemples:

- Agglutination par juxtaposition simple : la plus courante et la plus simple. Elle consiste à supprimer le trait d'union pour souder les deux mots. C'est le cas notamment des éléments formés avec auto (autogreffe, autoanticorps, mais auto-immune pour éviter un hiatus), ou aéro (aérotympanique), semi (semilunaire, semitendineux) ou ciné (cinédensitométrie), poly (polytraumatisme), etc. Mais aussi avec sous sauf sous-s... on écrira donc sousscapulaire mais subscapulaire, ou trans, y compris transs, comme dans transsudation.

- Agglutination par soudure avec contraction : disparition d'une lettre, généralement une voyelle, soit à la fin du premier mot soit au début du deuxième. Par exemple, contre-indication devient contrindication (je suis d'accord avec vous, c'est moche et ça accroche), mono-orchidie devient monorchidie, néo-arthrose se transforme en néarthrose.

- Agglutination avec doublement de consonne : la dernière lettre du premier mot et la première lettre du deuxième mot sont identiques. On écrira donc posttraumatique, posttransfusionnel (et si vous êtes comme moi, vous aurez du mal à laisser le deuxième " $t$ ").

D'une manière générale, on conserve le trait d'union lorsque la rencontre de deux voyelles change la prononciation, c'est-à-dire entre :

- " $a$ " et " $i$ "

- " a " et "u " 
- "e " et "u "

- " 0 " et " u

- " 0 " et " $i "$

Les pièges et difficultés qui jalonnent chaque texte médical renforcent la nécessité pour le traducteur de connaître le domaine, de se former et de lire la littérature médicale en particulier dans la langue cible pour se familiariser avec la terminologie et la syntaxe, et être ainsi capable de s'adapter à tout public. II doit en outre disposer des bons outils pour faire les recherches, à commencer par des dictionnaires spécialisés monolingues dans les différentes langues, mais aussi des ouvrages qui récapitulent les principes et les difficultés de la terminologie et de la traduction médicales, sur lesquels il pourra s'appuyer en cas de doute. Voici quelques pistes:

\section{Ouvrages indispensables}

Le domaine médical est vaste, et le traducteur se constituera sa propre bibliographie en fonction des sous domaines dans lesquels il est spécialisé. Cependant, deux ouvrages sont à mon sens absolument indispensables à tout traducteur médical, y compris au plus confirmé :

- CHEVALLIER Jacques, Précis de terminologie médicale, Maloine, 2015.

- QUÉRIN Serge, Dictionnaire des difficultés du français médical, Maloine, (nouvelle édition 2017).

\section{Quelques sites à connaître}

- Dictionnaire de l'académie de Médecine :

http://dictionnaire.academie-medecine.fr/ [les termes de la Terminologia Anatomica sont suivis de la mention (TA)].

- Dictionnaire de l'académie de Pharmacie : http://dictionnaire.acadpharm.org/w/Acadpharm:Accueil

- Biotop (terminologie médicale) : http://www.bio-top.net/

- Orphanet : http://www.orpha.net/consor/www/cgi-bin/index.php?lng=FR

- Vocabulaire de la santé : http://tinyurl.com/08gpq2h

- Portail Terminologique de Santé : http://www.hetop.org/hetop/

- Futura Santé (terminologie médicale) : http://tinyurl.com/melvq9r

nathalie@alp-traduction.fr 
Nathalie Renevier, traductrice-terminologue depuis 1997, est spécialisée dans la traduction de documents médicaux et juridiques, depuis l'anglais, l'italien et l'espagnol vers le français. Elle a traduit de nombreux ouvrages dans le domaine santé/médecine (éditions De Boeck, Larousse, Robert Laffont et Adverbum/Désiris). Elle intervient ponctuellement auprès des étudiants en traduction des universités de Toulouse et d'Angers (UCO) pour une première approche de traduction et terminologie médicale et assure de nombreuses formations dans le domaine de la traduction et de la terminologie, mais aussi en bureautique et en matière d'utilisation et de sécurité des réseaux sociaux, des blogs et d'IIIternet. Membre de la Société française des traducteurs et de la Société française de terminologie depuis plus de 10 ans, elle participe depuis 2010 aux travaux du Collège d'experts en terminologie dans le domaine de la santé et du social auprès du ministère de la Santé. À ses heures perdues, elle anime un blog d'actualité de la traduction et un autre qui propose des ressources pour les traducteurs et les terminologues.

http://ressources-alp-traduction.com/

http://www.alp-traduction.fr/blog/ 